\title{
Automatic diagnosis of tuberculosis disease based on plasmonic ELISA and color-based image classification
}

\begin{abstract}
Tuberculosis (TB) remains one of the most devastating infectious diseases and its treatment efficiency is majorly influenced by the stage at which infection with the TB bacterium is diagnosed. The available methods for TB diagnosis are either time consuming, costly or not efficient. This study employs a signal generation mechanism for biosensing, known as Plasmonic ELISA, and computational intelligence to facilitate automatic diagnosis of TB. Plasmonic ELISA enables the detection of a few molecules of analyte by the incorporation of smart nanomaterials for better sensitivity of the developed detection system. The computational system uses k-means clustering and thresholding for image segmentation. This paper presents the results of the classification performance of the Plasmonic ELISA imaging data by using various types of classifiers. The five-fold cross-validation results show high accuracy rate $(>97 \%)$ in classifying TB images using the entire data set. Future work will focus on developing an intelligent mobile-enabled expert system to diagnose TB in real-time. The intelligent system will be clinically validated and tested in collaboration with healthcare providers in Malaysia.
\end{abstract}

